\title{
The effectiveness of a cognitive behavioral exercise approach (CBEA) compared to usual care in patients with a Whiplash associated disorder: A quasi-experimental clinical trial
}

Jorge Hugo Villafañe ${ }^{\mathrm{a}, *}$, Davide Perucchini ${ }^{\mathrm{b}}$, Joshua A. Cleland ${ }^{\mathrm{c}}$, Cristina Barbieri ${ }^{\mathrm{a}}$, Fernanda de Lima e Sá Resende ${ }^{\mathrm{d}}$ and Stefano Negrini ${ }^{\mathrm{a}, \mathrm{e}}$

${ }^{a}$ IRCCS Don Gnocchi Foundation, Milan, Italy

${ }^{\mathrm{b}}$ Student of Physical Therapist, University of Brescia, Brescia, Italy

${ }^{c}$ Department of Physical Therapy, Franklin Pierce University, Concord, NH, L SA

d School of Physical Therapy, Alma Mater Studiorum, University of Bologn B logna, Italy

${ }^{e}$ Department of Clinical and Experimental Sciences, University of Bresein. Brescia, Italy

Abstract.

BACKGROUND: Whiplash Associated Disorders (WAD) is a bic syciosocial problem, education may be an essential part in the treatment and the prevention of chronic WAD. However, it is s ill unclear which type of educative intervention has already been used in WAD patients and how effective such interver tions a e.

OBJECTIVE: To examine the effectiveness of a cogniti e b havioral exercises approach (CBEA) for self-training of the neck relative to usual care in individuals with WAD in acut rilose.

METHODS: Forty-one patients, $65.9 \%$ female (me. $n \perp$ SD age: $41 \pm 11$ years), with WAD were recruited immediately after the accident (within 48 hours) and assigned acc rdi ing to patient choice to receive a CBEA self-training of the neck or usual care for 15 days. The primary outcome mea ve was pain intensity and disability as measured with the Neck Disability Index (NDI). Secondary outcome measures inc'u' 'ed the presence of headaches, dizziness, nausea, and difficulties with concentration and memory. Measurements were taken at pre-treatment, 2 weeks post-treatment and 4- and 12- weeks after the injury.

RESULTS: Patients receiving the CLEA intervention experienced a greater reduction in pain as compared to those receiving the usual care at the end as well as 4 a d 12 weeks after the intervention $(P<0.001)$, for the Neck Disability Index (NDI) decreased more in the CBEA than contre s o ver the 15 days and $\left(\mathrm{F}_{[3.0]}=552.383 ; P=0.001\right)$, and in both groups at all follow-up periods (all, $P=0.001)$.

CONCLUSIONS: This quas-experimental clinical trial provides evidence that a CBEA for self-training of the neck may be more beneficial in treating pain than usual care in patients with WAD. However, the CBEA had limited value in improving NDI. Future studies should include several therapists, a measure of a long-term outcomes and randomize patients to groups.

Keywords: Whiplash, cognitive-behavioural exercises, neck

\section{Introduction}

Whiplash associated disorders (WAD) consist a soft tissue or bony injury as a result of a swift acceleration

${ }^{*}$ Corresponding author: Jorge Hugo Villafañe, Regione Generala 11/16. Piossasco 10045, Italy. Tel.: +39 011 9065495/+39 339 5857563; Fax: +39011 9065495; E-mail: mail@villafane.it. and deceleration of the head and neck [1]. Whiplash associated disorders are a disabling condition and the patients symptoms may include neck pain, arm pain and headaches [1]. It has been reported that WAD continues to rise and the incidence has been reported to be approximately 300 per 100.000 individuals [2]. Although it has been reported that the majority of patients with WAD will experience a full recovery within a few 
months, it is estimated that approximately $50 \%$ will continue to experience pain and disability at a 1 -year follow-up [3]. The yearly cost associated with WAD is estimated to $\$ 3.9$ billion dollars in the United States an 13.4 billion dollars in Europe resulting in a substantial economic burden [4] Furthermore, it should also be recognized patients with WAD may experience signs of abnormal sensory processing and central sensitization leading to the development of chronic symptoms at long-term follow-up $[1,6]$.

In Italy, patients with WAD were commonly subjected to soft neck collar and painkillers as the first method of treatment following injury. Clinical practice guidelines have recommended rapid return to usual activity, education, manual therapy and therapeutic exercise as viable treatment approaches for reducing disability [5]. It has also been shown that patients with high baseline pain scores and disability have a poor prognosis [7]. Although number of interventions including exercise have been suggested as effective treatment options for the management of WAD [7,8], a recent systematic review suggested that there is inconclusive evidence that neck exercises are beneficial in the management of patients with WAD [9].

Patients with WAD often experience symptoms beyond physical impairments as a result of inappropriate pain behaviours and high levels of stress. It has be en suggested that it is essential to address both $\operatorname{cog} 11 \mathrm{iv}$ and behavioural manifestations in patients wiv in an attempt to maximize recovery [10]. $\int \mathrm{c}$ sse series of patients with WAD demonstrate tirt patients managed with a cognitive-behavioral â proach experienced a reduction of symptoms an a ril return of function [11]. A clinical trial comrarmo a CBT approach to a group receiving just the ercises [12] demonstrated those recivieing CB. . experienced significantly less pain at follow-up. The authors suggested that future studies should continue to examine the impact of CBT in patients with WAD and include larger sample sizes. The acute phase of WAD is relatively different from the chronic phase, since it includes either patients experiencing a full recovery in short time and patients prone to chronicity); one can wonder if a preventive approach combining the two best evidence based rehabilitation practices (exercises, within a cognitive-behavioural approach) can improve results in the short and long term of patients with WAD. Before proceeding to a randomised controlled trial, this hypothesis needs to be examined through observational data. Therefore the purpose of the current clinical trial was to examine the effects of exercises and a cogni- tive behavioural exercise approach (CBEA) compared to usual care for reducing pain and disability in a population with WAD in the acute phase (within 48 hours from injury).

\section{Methods}

\subsection{Design}

We conducted a quasi-experimental clinical trial. Informed consent was obtained from all participants and the protocol was approved by the Ethical Committee of IRCCS Don Gnocchi Foundation, Milan, Italy.

\subsection{Assignment of patients}

Forty-one patie $1 \mathrm{t}, 65.9 \%$ female (mean $\pm \mathrm{SD}$ age: $41 \pm 12$ years and $34.1 \%$ male (mean \pm SD age: 40 \pm 11 years), vi $b \mathrm{a}$ WAD as a result of a motor vehicle accider t were consecutively recruited within 48 hours of the in .ry from March 2013 to October 2013 at the Emer rer cy Unit of Istituto Clinico Città di Brescia, 3 escia, Italy. To be included in the study, the particirants had to be 18-70 years of age and needed to have a diagnosis WAD grades 1 and 2 according to the clinical classification of the Quebec Task Force of 1995 [1] as determined by an emergency room physician, had to have a score greater than 4 points at the $\mathrm{Vi}$ sual Analogue Scale (VAS) [13] without clinical signs of nerve root compression [14] and simultaneous concussion or other head trauma [15]. All patients underwent radiographic examination to exclude vertebral fractures [16].

According to usual care practices in Italy, each participating patient was prescribed a soft neck collar and painkillers to be used for a period between 7 and 14 days. Patients selectively chose to participate in one of 2 groups: an experimental group who received CBEA for self-training of the neck or a control group who received usual care intervention for 15 days. All outcome measures were collected at baseline, immediately following the intervention period, and 1- and 2-months following the end of the intervention.

\subsection{Sample size calculation}

The sample size and power calculations were performed with the ENE 3.0 software (GlaxoSmithKline C). Universidad Autónoma, Barcelona). The calculations were based on detecting a mean difference of $2 \mathrm{~cm}$ on 
a $10 \mathrm{~cm}$ visual analogue scale (VAS) assuming a standard deviation of $2 \mathrm{~cm}$, a 2-tailed test, an alpha level of 0.05 , and a desired power of $80 \%$. The estimated desired sample size was 16 individuals per group.

\subsection{Outcome measures}

\subsubsection{Current pain}

The primary outcome measure was neck pain intensity which was assessed with a VAS. The VAS is a $10 \mathrm{~cm}$ line anchored with a " 0 " at one end representing no pain and " 10 " at the other end representing the worst pain imaginable [9]. Pain was assessed at rest. The VAS was selected as the primary outcome measure based on its ability to detect changes and has a reported minimal clinically important difference (MCID) of $2.0 \mathrm{~cm}[17,18]$

\subsubsection{Neck disability index}

Disability was assessed using the Italian version of Neck Disability Index (NDI) [19], a 10-item selfadministered questionnaire. There are four items that relate to subjective symptomatology (pain intensity, headache, concentration, sleeping) and six items that relate to activities of daily living (lifting, work, driing, recreation, personal care, reading). Each questi in is scored on a 6-point scale ranging from 0 (no dis? 1 ity) to 5 (full disability), and these are adder to rether to make a total score ranging from 0 to 50 , which is interpreted as follows: 0 to $4=$ no dirat $4 i$ y, 5 to 14 $=$ mild disability, 15 to $24=$ modera e a sability, 25 to $34=$ severe disability, and greate th $n 34=$ complete disability. A blind assessor co verted these scores to a percentage.

\subsubsection{Common symptoms}

The patients were asked about the symptoms that they related to the whiplash injury. The symptoms assessed included neck pain, headache, dizziness, stiffness, nausea, shoulder and spine pain, anxiety, irritability, concentration problems and sleeplessness. We assessed the presence or absence of these symptoms (yes/no).

All outcomes measures were assessed pre-treatment, at 2 weeks post-treatment and 4 - and 12-weeks after the accident. The sequence of testing for the outcome measures was randomized among participants. The trial was designed according to the STROBE publishing guidelines [20].

\subsection{Intervention}

Guidelines for the management of whiplash [21] recommend that patients be provided educational material about WAD so all patients were provided with an educational booklet. The booklet was specifically created to improve the patient's beliefs about whiplash injuries and management strategies. The booklet provided information about the mechanism of injury, advice on suitable activities and reassurance about recovery, suggesting patients quickly get back to their normal activities of daily living and work, avoiding rest and prolonged immobilization [22].

\subsubsection{CBEA group}

Patients in this grou roceived information about whiplash, reassurance a but recovery and instructions about the importarc ot active self-training of the neck and a rapid ret ${ }^{-1} \mathrm{n}$ activities of daily living after a whiplash injury. 1 ll patients were prescribed a 15-day protoc 1 of ac.ive exercises beginning on the first day of treatmint under the supervision of a physiotherapist whic were to be continued at home; first while wearir $\mathrm{g}$ a neck collar according to the physician's prescripi. (that precluded end-of-range movement, and ofiered a gentle resistance to exercises), then without the collar. The protocol consisted in 7 neck movement exercises (rotation, side bending, bending and extension, neck retraction), 4 neck strengthening exercises (isometric, no movement exercise - bending, side bending and extension) and 3 shoulder movement exercises (flexion, rotation, extension). Patients were instructed to perform these exercises in the sitting position once a day. Movement exercises were designed to improve ROM of the neck. Patients were instructed to perform gentle and active movements up to a maximum range within their pain tolerance. The movements were repeated 10 times in each direction.

Neck strengthening exercises were designed to improve neck stability. Patients were instructed to perform a muscle contraction with the resistance of one hand for 10 seconds in each direction without feeling pain. Shoulder exercises were designed to improve ROM and strength of shoulder muscles. Patients were instructed to begin these exercises when they were no longer wearing the neck collar [5,7].

\subsubsection{Usual care group}

Patients in this group received usual care consisting of immobilization in a soft collar, the use of painkillers and a sick leave for a period between 7 and 14 days. 
In the case of persisting symptoms the general practitioner determined if the patient was to receive further treatment/medical examination. Patients were interviewed at pre-treatment and during follow-up periods.

\subsection{Statistical analysis}

Data were analyzed using SPSS version 19.0 (SPSS Inc, Chicago, IL), and conducted following an intention-to-treat analysis using the last value forward method. The results are expressed as means, standard deviations, and/or 95\% confidence intervals. The Kolmogorov-Smirnov test showed a normal distribution of the data. Potential differences in baseline demographic and clinical variables between groups were analyzed using independent Student t-tests for continuous data and chi-square of independence for categorical data. For the main outcome of the study, a $2 \times$ 3 repeat measures analysis of variance ANOVA was used to examine the effects of treatment on pain intensity and disability index at each measurement occasion as the dependent variable with group (CBEA, usual care) as the between-subjects variable and time (pretreatment, 2 weeks post-treatment and 4- and 12-weeks after the accident) as the within-subjects variable. The main hypothesis of interest was Group $\times$ Time inter $c$ tion. Post-hoc comparisons were conducted with $\mathbf{D}$ ferroni correction Between-groups effect sizus were calculated by using Cohen's d coefficient. An effect size greater than 0.8 was considered lare , round 0.5 moderate, and less than 0.2 small. Th atistical analysis was conducted at a $95 \%$ confid $\pi$ c level and a $P<$ 0.05 was considered statistically sirnificant.

\section{Results}

\subsection{Demographic data}

Fifty $(n=50)$ consecutive subjects with WAD were screened for eligibility criteria. Forty-one patients (mean \pm SD age: $41 \pm 11$ years; $65.9 \%$ female) satisfied all eligibility criteria, agreed to participate, and were assigned according to patient choice to the experimental $(n=25)$ or control $(n=16)$ group. The reasons for ineligibility included head trauma $(n=5)$, vertebral fractures, $(n=1)$, and clinical signs of nerve root compression $(n=3)$. Baseline features of both groups were similar for all variables (Table 1). There were no statistically significant differences in the re-

\begin{tabular}{|c|c|c|}
\hline \multicolumn{3}{|c|}{$\begin{array}{c}\text { Table } 1 \\
\text { Baseline demographics for both groups* }\end{array}$} \\
\hline & $\begin{array}{l}\text { CBEA group } \\
{[\text { no. }(\%)]} \\
(n=25)\end{array}$ & $\begin{array}{l}\text { Usual care group } \\
{[\text { no. }(\%)]} \\
(n=16)\end{array}$ \\
\hline Age (yrs) $($ mean \pm SD) & $40 \pm 12$ & $43 \pm 11$ \\
\hline \multicolumn{3}{|l|}{ Gender } \\
\hline Men & $7(28.0)$ & $7(43.8)$ \\
\hline Female & $18(72.0)$ & $9(56.2)$ \\
\hline $\begin{array}{l}\text { Neck pain intensity } \\
\text { (VAS, } 0-10 \text { ) }\end{array}$ & $6.7(1.1)$ & $6.2(1.0)$ \\
\hline $\begin{array}{l}\text { Neck disability index } \\
\quad(\text { NDI, } 0-100)\end{array}$ & $60.5(11.3)$ & $58.1(8.4)$ \\
\hline \multicolumn{3}{|l|}{ Pain killer } \\
\hline Yes & $22(88)$ & $21(81.3)$ \\
\hline No & $3(12)$ & $4(18.8)$ \\
\hline
\end{tabular}

*Data are expressed as means \pm standard deviation (SD); CBEA cognitive behavioral exercise appr ac.

Common symptoms pr.tment and 12 weeks later for both groups

\begin{tabular}{|c|c|c|c|c|}
\hline & \multirow{2}{*}{\multicolumn{2}{|c|}{$\begin{array}{l}\text { Pre-treatment } \\
(n=41)\end{array}$}} & \multirow{2}{*}{\multicolumn{2}{|c|}{$\begin{array}{c}12 \text { weeks after the } \\
\text { Accident }(n=41) \\
\text { Symptoms }\end{array}$}} \\
\hline & & & & \\
\hline & Yes $(\%)$ & $\operatorname{No}(\%)$ & Yes $(\%)$ & $\mathrm{No}(\%)$ \\
\hline \multicolumn{5}{|l|}{ Finge } \\
\hline CBEA & 100 & 0 & 0 & 100 \\
\hline Usual care & 56.3 & 43.8 & 46.2 & 53.8 \\
\hline \multicolumn{5}{|l|}{ Dizziness } \\
\hline CBEA & 60 & 40 & 4.3 & 97.5 \\
\hline Usual care & 68.8 & 31.3 & 0 & 100 \\
\hline \multicolumn{5}{|l|}{ Nausea } \\
\hline CBEA & 60 & 40 & 0 & 100 \\
\hline Usual care & 62.5 & 37.5 & 0 & 100 \\
\hline \multicolumn{5}{|c|}{ Difficulty with concentration } \\
\hline CBEA & 0 & 100 & 0 & 100 \\
\hline Usual care & 6.3 & 93.8 & 0 & 100 \\
\hline \multicolumn{5}{|l|}{ Insomnia } \\
\hline CBEA & 64.0 & 39.0 & 0 & 100 \\
\hline Usual care & 68.8 & 31.3 & 7.7 & 92.3 \\
\hline \multicolumn{5}{|l|}{ Neck stiffness } \\
\hline CBEA & 100 & 0 & 0 & 100 \\
\hline Usual care & 100 & 0 & 23.1 & 76.9 \\
\hline \multicolumn{5}{|l|}{ Neck pain } \\
\hline CBEA & 100 & 0 & 2.8 & 95.7 \\
\hline Usual care & 100 & 0 & 38.4 & 61.5 \\
\hline \multicolumn{5}{|l|}{ Back pain } \\
\hline CBEA & 56.0 & 54.0 & 0 & 100 \\
\hline Usual care & 50.0 & 50.0 & 7.7 & 92.3 \\
\hline \multicolumn{5}{|l|}{ Shoulder pain } \\
\hline CBEA & 76.0 & 24.0 & 8.7 & 91.3 \\
\hline Usual care & 68.8 & 31.2 & 7.7 & 92.3 \\
\hline
\end{tabular}

CBEA: cognitive behavioral exercise approach.

ported frequency of symptoms after the accident, for any demographics or outcomes at baseline. No adverse events were reported during or after the application of the treatment, and none of the patients started taking new medications during the study. 
J.-H. Villafañe et al. / Management of patients with Whiplash

$\begin{array}{cccccc}\text { Table } 3 & & \\ \text { Treatment type post-treatment and } 12 \text { weeks later for both groups }\end{array}$

CBEA: cognitive behavioral exercise approach.

\subsection{Common symptoms}

Pre-treatment, $82.9 \%$ of the total patients reported severe headache, $63.4 \%$ reported severe dizziness, $61.0 \%$ reported severe nausea, $100 \%$ reported severe neck pain and $100 \%$ reported severe neck stiffness. After 12 weeks, $16.7 \%$ of the patients reported severe headache, $2.8 \%$ reported severe dizziness, $0 \%$ reported severe nausea, $16.7 \%$ reported severe neck pain and $8.3 \%$ reported severe neck stiffness (Table 2).

\subsection{Frequency of additional treatment}

At two weeks after the injury, 53.7\% of th ratients used painkiller and $36.6 \%$ of the patie $1 \mathrm{t}$ ued a neck collar. $34.1 \%$ of the patients used sic: leave and $56.4 \%$ of the patients used exercises ( $\mathrm{Ta}$ le 3 ).

\subsection{Neck pain intensity (VAD)}

For the VAS, the ANUVA revealed a significant effect of time $\left(\mathrm{F}_{[3.0]}=181.335 ; P=0.001\right.$, partial eta $=$ $0.842)$ and for the group-by-time interaction $\left(\mathrm{F}_{[3.0]}=\right.$ $13.188 ; P=0.001$, partial eta $=0.279$ ) for pain intensity. The post hoc analysis revealed significant within group differences for the CBEA $(P=0.001)$, but not for the usual care group $(P=1.0)$. We found significant differences between the pretreatment and followup periods in the CBEA and Usual care group (all, $P=$ 0.001) (Table 4). Between-groups mean differences post-intervention was $-2.5(95 \% \mathrm{CI} ;-3.9,-1.1)$, the

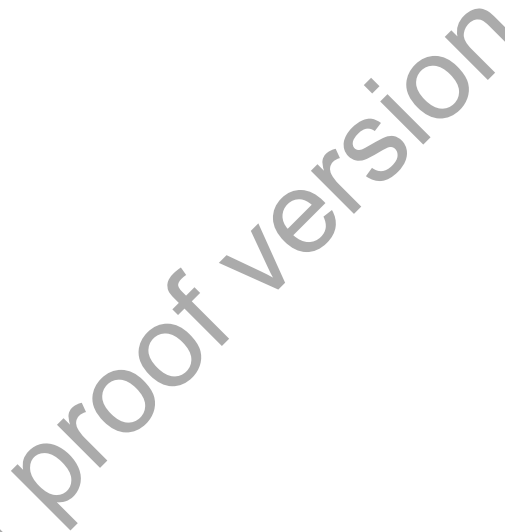
4 week follow-up was $-2.7(95 \% \mathrm{CI} ;-4.0,-1.5)$ and the 12 week follow-up was $-1.1(95 \% \mathrm{CI} ;-1.9,-0.4)$ for VAS. Between-groups effect sizes were small at post-treatment and follow-up periods (all, $d<0.2$ ).

For the NDI there was a significant effect of time 
6

and group-by-time $\left(\mathrm{F}_{[3.0]}=13.409 ; P=0.001\right.$, partial eta $=0.283$ ), interactions (Table 4$)$. All participants in both groups demonstrated changes (decreases) over the 15 days and follow-up periods (all, $P=$ 0.001). There was significant difference between the groups at post-treatment and follow-up periods (all, $P=0.001)$. Between-groups effect sizes were small at post-treatment period and follow-up periods (all, $d<$ $0.2)$.

\section{Discussion}

This quasi-experimental trial examined the effects of a CBEA program including patient education and self-training of the neck musculature in a patient population with WAD. The results demonstrated that those patients who received the CBEA experienced significantly greater improvements in pain and disability compared to those who received usual care. It is interesting to note that the between group differences for pain improvements and the lower bound estimate of the 95\% confidence interval exceeded the reported MCID of $2.0 \mathrm{~cm}$ for the VAS [17,18]. We believe this provides preliminary evidence to support the use of a CBEA in the management of patients with WAD, supporting the importance of a future randomized controlled trial, a ia providing preliminary results which may assist vild future power analysis.

The current results support the hypothesi of Soderlund et al. [10] who has suggested that arultimodal approach including biopsychosocial co mponents may be beneficial in reducing pain aru aisability as well as preventing chronicity in a pation population with WAD. Furthermore the currest esults support those of Adams et al. [23] and Sulivan et al. [24] who both found that a multimodai $p h y$ sical therapy program that included a psychosocial intervention resulted in greater function and a more rapid return to work. However, these 2 studies were not experimental and hence a cause and effect relationship could not be. Additionally, our results support those of Schnabel et al. [25] who randomly assigned a group of patients to wear a collar or participate in an exercise program. At the end of 6-weeks patients in the exercise group experienced significantly greater improvements in both pain and disability. Perhaps immobilization with a soft-collar should not be prescribed to patients after a WAD unless it is medically necessary (cervical instability).

It should also be noted that we started treatment of the patients in the study within 48 hours of their injury. 


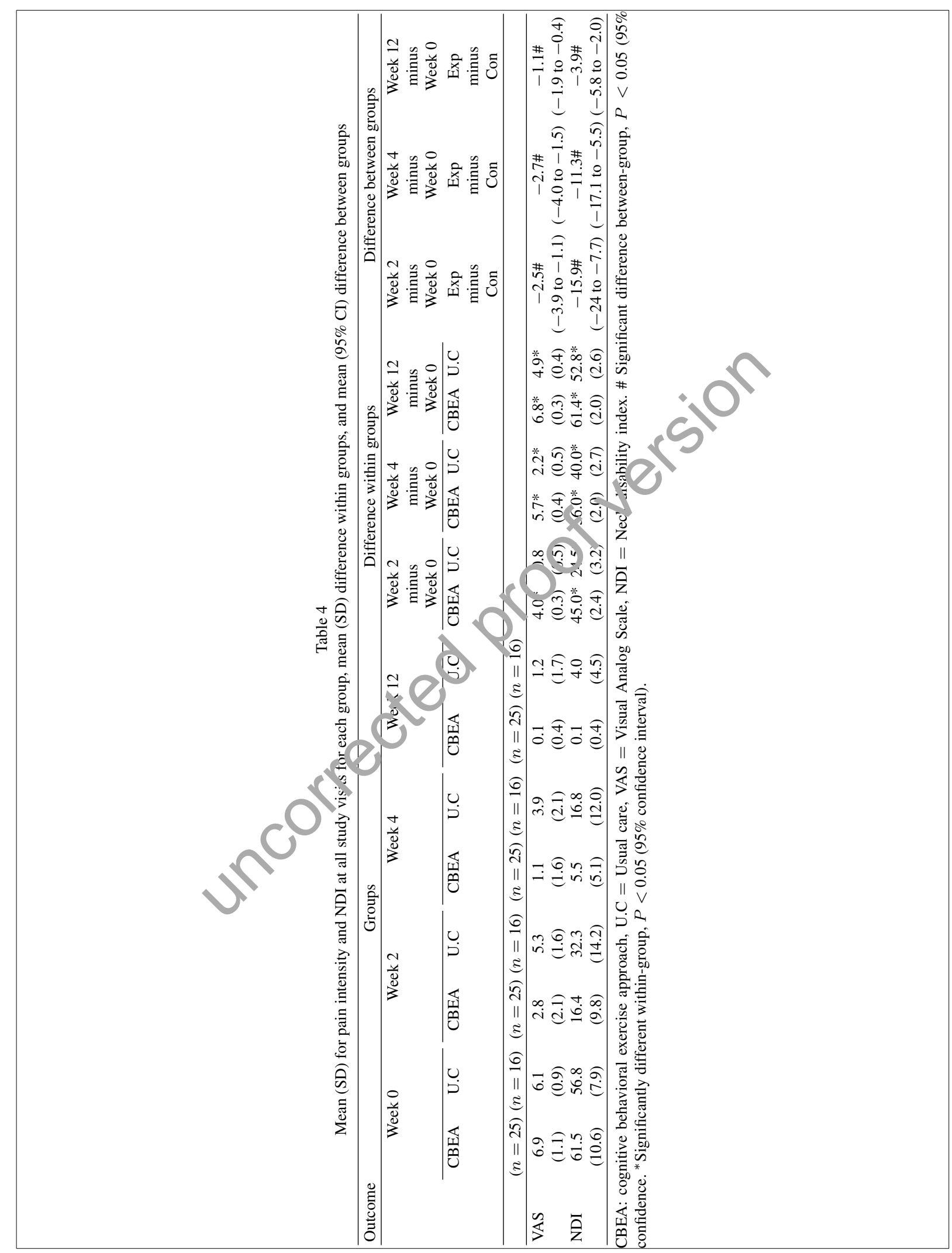


So it is possible that the early initiation of exercise might have assisted with reducing the patient's symptoms in the CBEA group. Rosenfeld et al. [26] randomly assigned patients to receive an active intervention or usual care. Additionally, individuals in both groups started treatment either quickly after the WAD or more than 2 weeks after the injury. The results demonstrated that if the active group started treatment quickly (less than 96 hours) then they experienced significantly greater reductions in pain. Furthermore, in an additional analysis, these same authors [4] demonstrated that the early initiation of active treatment reduced the overall costs associated with the WAD. Perhaps this is the reason why patients in the current study exhibited improvement. Future studies should further examine the timing of treatment.

It is also possible that some would argue that it might not be the specific exercises we prescribed that resulted in reductions in pain but it could also be that education to stay active might be just as beneficial. However, Gwendolijine et al. [27] randomly assigned patients with a WAD to receive exercise prescribed by a physical therapists or treatment provided by a general practitioner which consisted of education on graded activity. Similar to our study, the results demonstrated that both groups got better but the group that received exercise under the direction of a physical therapists expe 1enced significantly greater pain reductions at the 0 year follow-up period.

There are a number of limitations to this stu ly that must be considered. Only 1 therapist per orned all interventions so the generalizability of he results may be limited. The follow-up period $v$ an limited to 12 weeks and therefore we cannot ce ce tain if the pain reduction lasted beyond that tinc dditionally, patients self-selected which group thry wanted to participate in. It might have been that the re are other characteristics associated with individuals who selected the exercise group that might have been responsible for the reductions in pain. We also did not have a true control group. Future randomized clinical trials should include a control group, include multiple therapists, randomize patients to treatment groups, and collect data at a longterm follow-up.

\section{Conclusion}

This quasi-experimental clinical trial provides the first evidence that the CBEA approach was more beneficial in reducing pain than usual care in this popula- tion of patients with WAD. However, the CBEA had limited value in improving disability. Patients in the CBEA also experienced significantly better for most secondary outcomes compared to usual care. Future studies are needed to further examine the effectiveness of the CBEA for the management of patients with WAD.

\section{Acknowledgements}

Jorge H. Villafañe, Davide Perucchini, Joshua Cleland, Cristina Barbieri, Stefano Negrini. A signed letter of permission from each person are attached.

\section{Conflicts of interest}

No conflicts of nterest.

\section{Referen}

S suzer WO, Skovron ML, Salmi LR, et al. Scientific monograph of the Quebec task force on whiplash-associated disorders: redefining "whiplash" and its management. Spine (Phila Pa 1976) 1995; 15; 20(8 Suppl): S1-73.

[2] Holm LW, Carroll LJ, Cassidy JD, et al. The burden and de terminants of neck pain in whiplash-associated disorders after traffic collisions: results of the bone and joint decade 2000 2010 task force on neck pain and its associated disorders. J Manipulative Physiol Ther 2009; 32(2 Suppl): S61-9.

[3] Carroll LJ, Holm LW, Hogg-Johnson S, et al. Course and prognostic factors for neck pain in whiplash-associated disorders (WAD): results of the bone and joint decade 2000-2010 task force on neck pain and its associated disorders. J Manip ulative Physiol Ther 2009; 32(2 Suppl): S97-107.

[4] Rosenfeld M, Seferiadis A, Gunnarsson R. Active Involvement and Intervention in Patients Exposed to Whiplash Trauma in Automobile Crashes Reduces Costs. A Randomized, Controlled Clinical Trial and Health Economic Evalua tion. Spine (Phila Pa 1976) 2006; 31: 1799-804.

[5] Scholten-Peeters GGM, Bekkering GE, Verhagen AP, et al Clinical practice guideline for the physiotherapy of patients with whiplash-associated disorders. Spine 2002; 27: 412-22.

[6] Kamper SJ, Rebbeck TJ, Maher CG, et al. Course and prog nostic factors of whiplash: a systematic review and metaanalysis. Pain 2008; 138: 617-29.

[7] Motor accidents authority: guidelines for the Management of Acute Whiplash-Associated Disorders for Health Profession als. 2nd Edition 2007.

[8] Drescher K, Hardy S, MacLean J, et al. Efficacy of Postu ral and Neck-Stabilization Exercises for Persons with Acute Whiplash-Associated Disorders: A Systematic Review. Phys iother Can 2008; 60: 215-23.

[9] Parazza S, Vanti C, O'Reilly C, et al. The relationship be tween cervical flexor endurance, cervical extensor endurance, VAS, and disability in subjects with neck pain. Chiropr Man Therap 2014 Mar 3; 22(1): 10. 
10] Söderlund A. The role of educational and learning approaches in rehabilitation of whiplash-associated disorders in lessening the transition to chronicity. Spine 2011; 36(25 Suppl): S2805 .

[11] Söderlund A, Olerud C, Lindberg P. Acute whiplashassociated disorders (WAD): the effects of early mobilization and prognostic factors in long-term symptomatology. Clin Rehabil 2000; 14: 457-67.

[12] Söderlund A, Lindberg P. Cognitive behavioural components in physiotherapy management of chronic whiplash associated disorders (WAD) - a randomised group study. Physiotherapy Theory and Practice 2001; 17: 229-38.

13] Price DD, McGrath PA, Rafii A, et al. The validation of visual analogue scales as ratio scale measures for chronic and experimental pain. Pain 1983; 17: 45-56

14] Kettner NW, Guebert GM. The radiology of cervical spine injury. J Manipulative Physio Ther 1991; 14: 518-26.

15] Evans RW. Some observations on whiplash injuries. Neurol Clin 1992; 10: 975-97.

16] Borchgrevink GE, Smevik O, Nordby A, et al. MR imaging and radiography of patients with cervical hyperextensionflexion injuries after car accidents. Acta Radiol 1995; 36: $425-$ 8.

17] Emshoff R, Bertram S, Emshoff I. Clinically important difference thresholds of the visual analog scale: a conceptual model for identifying meaningful intraindividual changes for pain intensity. Pain 2011; 152: 2277-82.

[18] Farrar JT, Pritchett YL, Robinson M, et al. The clinical im portance of changes in the 0 to 10 numeric rating scale for worst, least, and average pain intensity: analyses of data from clinical trials of duloxetine in pain disorders. J Pain 2010; 11: 109-118
[19] Monticone M, Ferrante S, Vernon H, et al. Development of the Italian Version of the Neck Disability Index: cross-cultura adaptation, factor analysis, reliability, validity, and sensitivity to change. Spine (Phila Pa 1976) 2012; 37: E1038-44.

[20] von Elm E, Altman DG, Egger M, et al. The Strengthen ing the Reporting of Observational Studies in Epidemiology [STROBE] statement: guidelines for reporting observationa studies. Gac Sanit 2008; 22: 144-50.

[21] Lindsay GM, Mior SA, Côté P, et al. Patients' Experiences With Vehicle Collision to Inform the Development of Clinical Practice Guidelines: A Narrative Inquiry. J Manipulative Physiol Ther 2016 Mar-Apr; 39(3): 218-28.

[22] McClune T, Burton A.K, Waddell G. Whiplash associated disorders: a review of the literature to guide patient informa tion and advice Emerg Med J 2002; 19: 499-506.

[23] Adams H, Ellis T, Stanish WD, et al. Psychosocial factor related to return to work following rehabilitation of whiplash injuries. J Occup Rehabil 2007: 17: 305-15

[24] Sullivan MJ, Adams H, R ode izer T, et al. A psychosocial risk factor-targeter in er vention for the prevention of chronic pain and dis a bu following whiplash injury. Phys Ther 2006; 86: 8- 8

[25] Schnabel M, Fu rari ., Vassiliou T, et al. Randomised, controlled outc on sudy of activemobilisation compared with collar the ap for whiplash injury. Emerg. Med. J 2004; 21 $305-10$.

[26] Rc. anfeld M, Gunnarsson R, Borenstein P. Early intervention in hiplash-associated disorders: a comparison of two treat ner c protocols. Spine (Phila Pa 1976) 2000; 25: 1782-7.

7] Scholten-Peeters GG, Neeleman-van der Steen CW, van de Windt DA, et al. Education by general practitioners or education and exercises by physiotherapists for patients with whiplash-associated disorders? A randomized clinical trial Spine (Phila Pa 1976) 2006; 31: 723-31. 